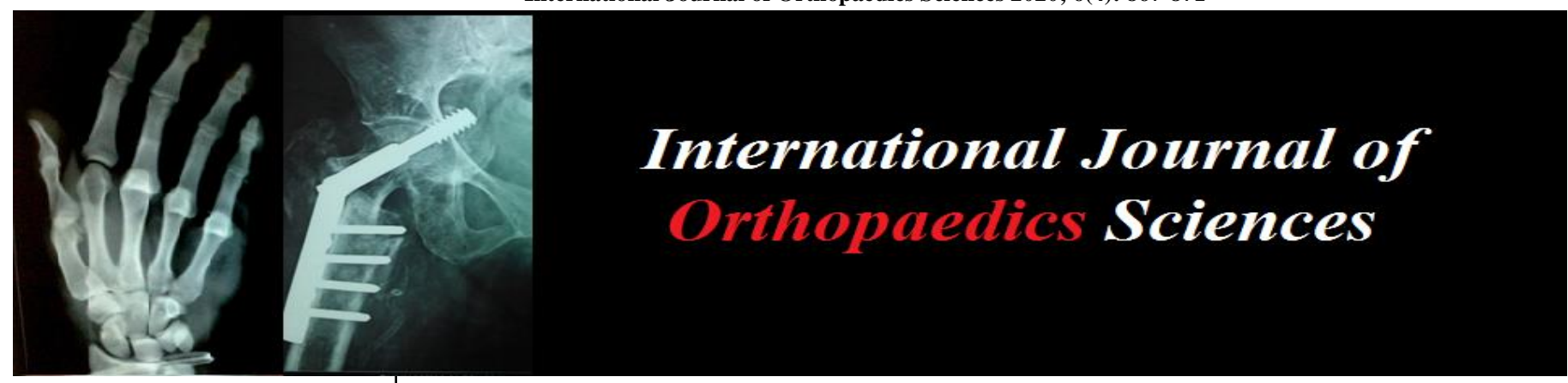

E-ISSN: 2395-1958

P-ISSN: 2706-6630

IJOS 2020; 6(4): 867-871

(C) 2020 IJOS

www.orthopaper.com

Received: 01-08-2020

Accepted: 05-09-2020

Dr. Avinash Gundavarapu Yashoda Superspeciality

Hospital, Rajbhavan road,

Hyderabad, Telangana, India

Dr. Santhosh Kumar M

Yashoda Superspeciality

Hospital, Rajbhavan road,

Hyderabad, Telangana, India.

Dr. Vishal Singh

Dhanwantri Hospital, Jaipur,

Rajasthan, India

Dr. Prashant Kumar Mishra

Yashoda Superspeciality

Hospital, Rajbhavan road,

Hyderabad, Telangana, India.

Corresponding Author:

Dr. Avinash Gundavarapu

Yashoda Superspeciality

Hospital, Rajbhavan road,

Hyderabad, Telangana, India

\section{Study of functional outcome following arthroscopic anatomical ACL reconstruction using autologous hamstring graft}

\author{
Dr. Avinash Gundavarapu, Dr. Santhosh Kumar M, Dr. Vishal Singh and \\ Dr. Prashant Kumar Mishra
}

DOI: https://doi.org/10.22271/ortho.2020.v6.i4m.2430

\section{Abstract}

Background: Our study aim is to assess the functional outcome of arthroscopy assisted anatomical reconstruction of ACL with autologous hamstring graft using IKDC score.

Methodology: Our study is a prospective observational study conducted in 40 patients, all patients between age group of 20-40 yrs, both male and females with ACL injury, admitted to Yashoda Superspeciality hospital, between April 2016 and May 2017 (14 months) and fulfilling inclusion and exclusion criteria, treated surgically with Arthroscopic anatomic ACL reconstruction using autologous hamstring graft were included as the subjects for study. Case selection was done according to.

Results: A total of 40 patients, 32 male and 8 female with 26 right knee and 14 left knee involvement with ACL tear were operated. The IKDC score reflected highly significant improvement in the stability, mainly rotational and anterior stability when preoperative score were compared with the score of most recent follow up i.e. 6 months. Pre-operative mean of 44.43+3.630 SD and Post-operative mean was 88.53+3.994 SD. The average Pre-operative mean was 44.525 and average Post-operative mean was 88.525 , with an average mean increase in score was 44 , with significant $\mathrm{P}$ value $<0.01$ using IKDC score.

Interpretation and conclusion: Anatomical ACL reconstruction is excellent technique for treating patients with ACL tear who are especially involved in the activities which involve pivoting most of the times in their life time for e.g. Athletes.

Keywords: Arthroscopy, anatomical ACL reconstruction, IKDC score

\section{Introduction}

Anterior cruciate ligament (ACL) is one of the common sports injuries seen by orthopedic surgeons and is the most common complete ligamentous injury in the knee. The anterior cruciate ligament is a band of dense connective tissue which runs from the posteromedial aspect of the intercondylar notch on the lateral femoral condyle towards anteriorly, medially and distally ${ }^{[1]}$. The narrowest part of the ACL is at midsubstance level $\left(35 \mathrm{~mm}^{2}\right)$. It is a major knee ligament to stabilize the joint movement against anterior tibial translation and rotational loads ${ }^{[1,2]}$. A recent systemic review by Hwang et al. showed that the ACL tibial foot print is $15 \mathrm{~mm}$ anterior to the ACL and two-fifth of medial-lateral width of the interspinous distance with the majority of the ACL fibres attaching anterior to the posterior margin of lateral meniscus ${ }^{[3]}$. The purpose of ACL reconstruction is to restore normal stability, and to protect the knee from further injury ${ }^{[5]}$. Anatomic ACL reconstruction can be defined as the functional restoration of the ACL to its native dimensions, collagen orientation, and insertion sites ${ }^{[4]}$. Anatomic placement of an anterior cruciate ligament (ACL) graft is critical to the success and clinical outcome of ACL reconstruction. Non-anatomic ACL graft placement is the most common technical error leading to recurrent instability following ACL reconstruction [5, 6] results in limited range of motion, higher than physiologic graft tension, and ultimately graft failure ${ }^{[7,8]}$. A vertically oriented ACL graft may resist the motion of anterior tibial translation, but may fail to control the combined motions of anterior tibial translation and internal rotation which occur during the pivot-shift phenomenon ${ }^{[9]}$. The purpose of this study is to assess the function of arthroscopy assisted anatomical ACL reconstructed knee in patients who underwent autologous hamstring graft reconstruction. 
The functional outcome is measured using International Knee documentation committee (IKDC).

\section{Material and Methods}

Our study is a Prospective Observational Study conducted in Yashoda Superspeciality Hospital, Rajbhavan road, Hyderabad. A total of 40 patients (both male and female) with ACL injury between age group of 20-40yrs admitted to Yashoda Superspeciality hospital, between April 2014 and May 2015 (14 months) were treated surgically with Arthroscopic anatomic ACL reconstruction using autologous hamstring graft after obtaining informed consent.

\subsection{Inclusion Criteria}

1. Clinical / radiological / arthroscopic evidence of anterior cruciate Ligament injury/deficiency, which is symptomatic even after conservative therapy of adequate duration.

2. Young and middle aged, active, motivated patients with future interest in professional / recreational sports aiming for active life style.

3. The acute inflammatory phase of the injury has subsided and full range of motion and good quadriceps strength has been regained with no extensor lag (usually after 4-6 weeks of injury)

4. Patients with regular follow-up visits to outpatient department.

\subsection{Exclusion Criteria}

1. Patients with bilateral ACL tear or associated periarticular fractures or fractures of lower limb and spine.

2. Patients with any other associated ligament injuries of the Knee (tear of posterior cruciate ligament, medial and lateral collateral ligament requiring treatment)

3. Patients with open physis.

4. Patients with local skin infections.

\subsection{Study Procedure}

Fourty Patients with Complete ACL injury, treated surgically with Autologous hamstring graft Arthroscopically, and with regular follow-up visits were included in this study. The study period was of 14 months. A detailed history and clinical examination was be done. The following specific tests were performed for diagnosing anterior cruciate ligament deficiency: Lachman test, Anterior drawer test, Pivot shift test and followed by preoperative IKDC Subjective Evaluation. Injuries to the associated structures were assessed by performing the following clinical tests:- Valgus / Varus stress test (for collateral ligaments), McMurray's test (for menisci), Posterior drawer test (for posterior cruciate ligament), Reverse pivot shift test (for Posterolateral complex). Routine skiagram of both knees in standing position in anteroposterior view and lateral view of the affected knee were taken and lateral view in $45^{0}$ of flexion of the affected knee was also done. MRI of the knee was done in cases with equivocal clinical findings. Diagnostic arthroscopic examination of the knees was used as last resort for patients with negative or equivocal clinical and radiological findings but consistent symptoms suggesting anterior cruciate ligament deficiency. After obtaining written informed consent, patients with established ACL injury were taken up for Arthroscopic Anatomic ACL reconstruction using autologous hamstring graft. Functional evaluation of the reconstructed ACL will be done using IKDC score.

\section{Post -Operative Care and Follow Up}

Post-operatively patients were placed in knee brace. Partial weight bearing with crutches (50\% of the body weight) was allowed for one month. Mobilization was started immediately, giving priority to the recovery of full extension. CPM was used to help flexion. Active static quadriceps exercises and ankle pumps were started as soon as the patient recovered from anesthesia. The crutches are maintained until quadriceps control was reestablished typically about 4-6 weeks. Standard ACL reconstruction rehabilitation protocol was then followed. Non cutting, Non-pivoting sports was allowed after 3 months. Cutting and pivoting sport activities were started by 6 months. All knees were examined before surgery, in the operating room, immediately after the procedure and at one, two, four, six months. Ability to bear weight (graded as full, partial, or impossible) was assessed postoperatively and at two, four and six months. Difficulty with squatting (assessed as no problem flexing the knees greater than or equal to 90 degrees, ability to flex the knees greater than or equal to 90 degrees with slight difficulty, unable to flex the knees greater than or equal to 90 degrees, or unable to squat) will be assessed at the preoperative, four and six month visits. Results were documented at two weeks, one, two, four and six months. In our study we used IKDC Score in order to evaluate the rehabilitation progress of patients with ACL reconstruction.

\section{Results}

Out of 40 patients with ACL tear in our study, 32 were males $(80 \%)$ and 8 were females $(20 \%)$. All the patients were between $20-40$ yrs of age.

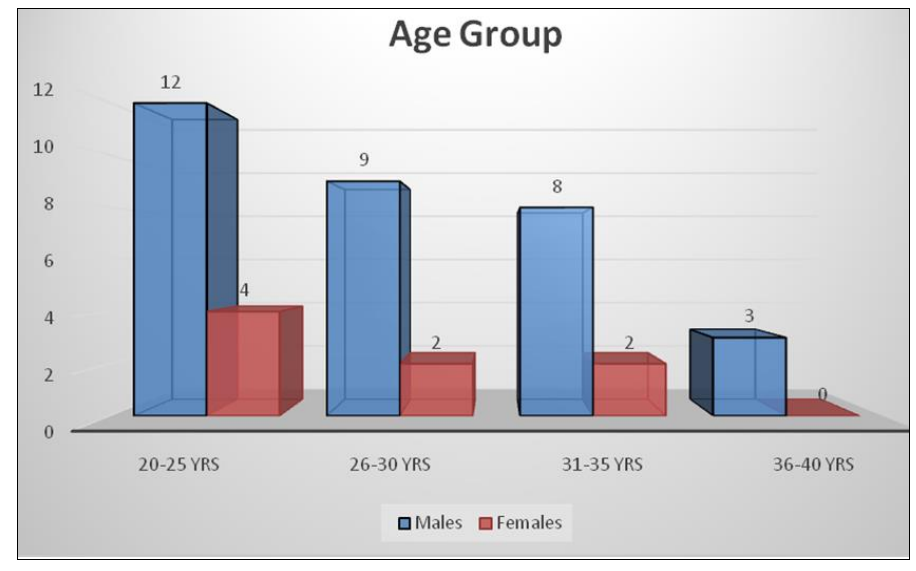

Graph 1: Age and Sex Distribution 
In our study $40 \%$ of cases were in the age group of 20-25 years, followed by $27 \%$ cases in $25-30$ years. Both Males and females predominantly belonged to age group 20-25 years. The mean age of patients in our study was 28.4 years. The Ratio of male to female patients was 4: 1 With right to left ratio of 1.86: 1. The duration of symptoms in our study ranged from day 0 to 11 months, Out of which $45 \%$ of the patients were operated between 2 to 6 months. Road traffic accidents accounted for $30 \%$ ( 12 cases) of patients while $60 \%$ (24 cases) were due to sports injury. Injury sustained during activities of daily living accounted for $10 \%$ (4 Cases). Graph 2 -

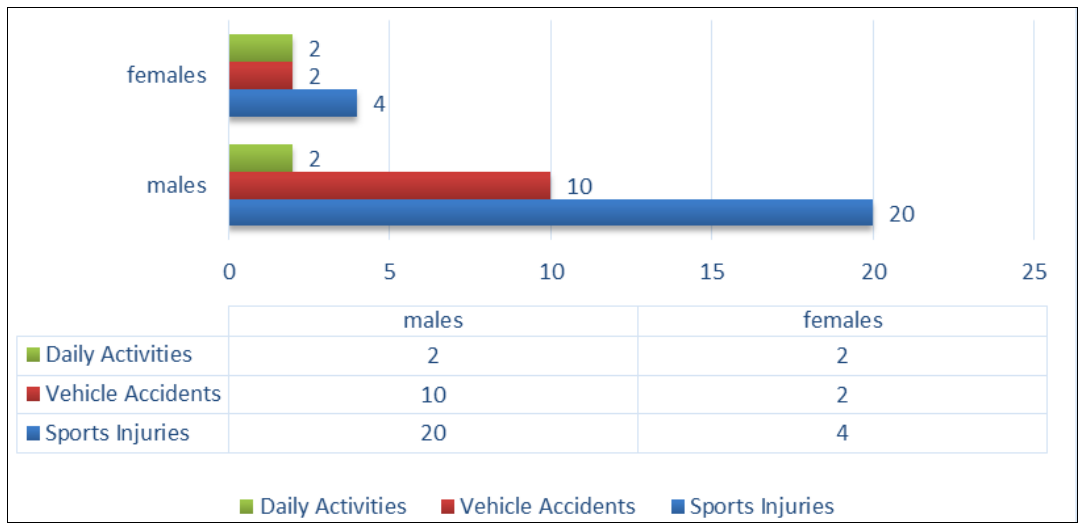

Graph 2: Injury sustained during activities of daily living accounted

Majority of the patients $60 \%$ (24 cases) presented with a feeling of giving way of the knee, during routine work and guarded walking due to apprehension, usually without pain. $40 \%$ (16 cases) patient had a feeling of giving way during heavy laborer or playing usually with pain. The most common clinical finding associated in the patients of ACL tear was effusion, which was present in $70 \%$ (28 patients) of cases. The joint line tenderness was present in $60 \%$ (16 patients) of the cases. There were $30 \%$ cases (12 patients) with flexion < 100 degrees. There were only two cases (5\%) who had Flexion < 10 degrees. Manual knee laxity test were performed in all cases before ACL reconstruction in which Anterior Drawer Test, Lachman test and Pivot shift test were positive in $100 \%$ of cases. Results of Manual knee laxity test after anatomical ACL reconstruction showed $82.5 \%$ cases were Lachman test negative, $12.5 \%$ were Grade 1 and only $5 \%$ were Grade 2. Results of Anterior Drawer Test after ACL reconstruction were $85 \%$ were negative, $10 \%$ were Grade 1 and $5 \%$ were Grade 2. Results of Pivot shift test after double bundle ACL reconstruction were $72.5 \%$ were negative, $20 \%$ were Grade 1 and $7.5 \%$ were Grade 2 . There were no cases of severe instability in our series with Grade 3 for Lachman test, Anterior drawer test and Pivot shift test.

The results of Pre-operative and Post-operative Lachman test were statistically evaluated and the mean of pre-operative Lachman test was $2.43+0.675 \mathrm{SD}$ and post-operative lachman test has $0.23+0.530 \mathrm{SD}$ with significant $\mathrm{p}$ value $<$ 0.01 .

Table 1: Pre-operative findings.

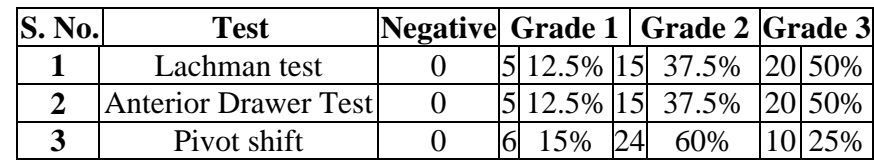

Table 2: Post-operative findings

\begin{tabular}{|c|c|c|c|c|c|c|c|c|c|}
\hline S. NO & Test & \multicolumn{2}{|c|}{ Negative } & \multicolumn{2}{c|}{ Grade 1 } & \multicolumn{3}{|c|}{ Grade 2 } & \multicolumn{2}{|c|}{ Grade 3 } \\
\hline 1 & Lachman Test & 33 & $82.5 \%$ & 05 & $12.5 \%$ & 02 & $5 \%$ & 00 & $0 \%$ \\
\hline 2 & $\begin{array}{c}\text { Anterior Drawer } \\
\text { Test }\end{array}$ & 34 & $85 \%$ & 04 & $10 \%$ & 02 & $5 \%$ & 00 & $0 \%$ \\
\hline 3 & Pivot shift test & 29 & $72.5 \%$ & 08 & $20 \%$ & 03 & $7.5 \%$ & 00 & $0 \%$ \\
\hline
\end{tabular}

The results of Pre-operative and Post-operative Anterior Drawer test were statistically analyzed and the mean of preoperative Anterior Drawer Test was $2.40+0.672$ SD and Post-operative was $0.20+0.516 \mathrm{SD}$ with significant $\mathrm{p}$ value $<0.01$. Similarly the results of preoperative Pivot shift test (PST) and Post-operative Pivot shift test were statistically analysed. The mean preoperative PST was $2.10+0.632$ SD and post-operative was $0.35+0.622$ with significant $p$ value $<0.01$. The results of IKDC Score were statistically analyzed using the paired-t test at $5 \%$ level of significance, the mean preoperative score was $44.53+3.630 \mathrm{SD}$ and post- operative mean score was $88.53+3.994 \mathrm{SD}$ with significant $\mathrm{p}$ value < 0.01 .

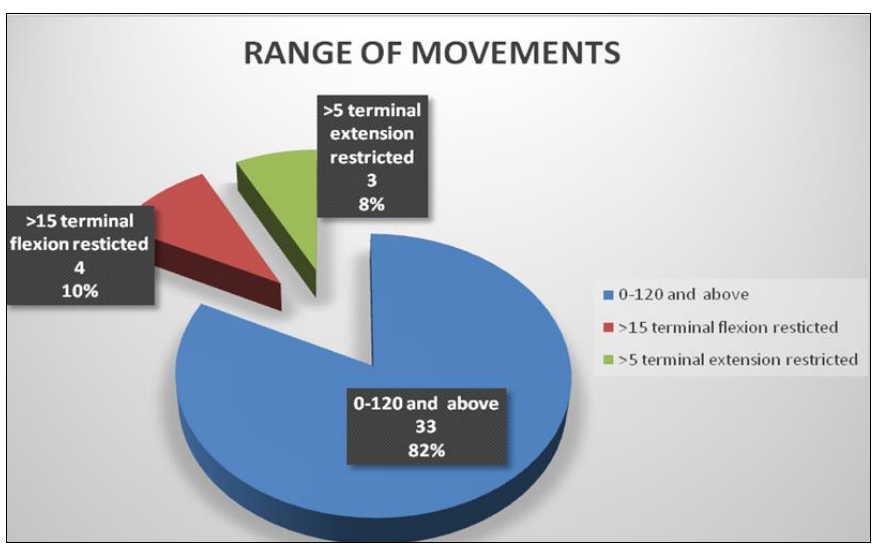

Graph 3: Range of Motion.

No major complications were seen in our series except superficial infection in 3 cases $(7.5 \%) .3$ cases $(7.5 \%)$ had difficulty in regaining the motion. There were no cases in our series which had graft avulsion, Graft impingement. There were 3 cases $(7.5 \%)$ of superficial infection which were all treated and responded well to intravenous antibiotics.

\section{Discussion}

Most of our patients in our series - 16 cases (40\%) were in the age group 20 - 25 years followed by $27 \%$ (11 cases) in $25-$ 30 years age group. Brig VP Pathania, Lt Col GR Joshi et al. [10] in there series showed the maximum number of patients 
10 cases $(40 \%)$ were grouped between $25-30$ years of the age. The age of the patients ranged from $20-40$ years with the mean age of 28.4years in our study and A.J. Johnson, E. Eriksson, T. Haggmark and M.H. Pope (1984) in their series had patients whose age ranged from 17 to 48 years with the mean age of 26.3 years.

Takeshi Muneta et al. ${ }^{[11]}$ in their study indicated that double bundle ACL reconstruction via 4 stranded ST is superior to the single bundle technique with regard to anterior and rotational stability. Freddie $\mathrm{Fu}$ and Christian letterman et al ${ }^{12}$ suggest that anatomical ACL double bundle reconstruction may result in better restoration of in vivo kinematics of knee joint and can improve clinical outcomes in ACL surgery.

The ratio of the right knee to left knee in this series was 1.8:1 and A.J. Johnson, E. Eriksson et al. (1984) in their study had right to left knee involvement ratio of 1.2: 1 .

Sports injuries $(60 \%)$ predominates as mode of injury in our series and William G Clancy, Jr Devon A. Nelson, Bruce Reider and Rajesh G Narechania (1982) had 88\% of sports related injuries.

The major presenting complaint was guarded walking. $50 \%$ (20 cases) presented with a feeling of giving way of the knee during routine work with no or minimal pain or guarded walking. In rest of the cases $30 \%$ (12 cases) patient had a feeling of giving way with pain during heavy labor or sports activity. However, they all had various associated complaints like Effusion (70\%), Joint line tenderness (40\%), difficulty in climbing staircase and difficulty in squatting. R.J. Johnson, E. Eriksson, T. H. Haggmrk and M.H. Pope (1994) ${ }^{[13]}$ in their study 73 patients $(83.9 \%)$ complained of a feeling of giving way of the knee, associated with joint effusion (92\%).

All the patients were evaluated symptomatically by IKDC score, clinically and radiologically at 2 weeks, one month, 3 months, 6 months, and statistical analysis was done. The average preoperative IKDC score was $44(39-52)$. Average post-operative Lysholm score at 6 months follow up was 88 $(80-94)$. The average increases in points were 44 . After the average follow up of 6 months above 90\% patients were satisfied with their result.

In our study $82.5 \%$ (33 cases) were negative Lachman test, The mean of pre-operative Lachman test was $2.43+0.675$ SD and post-operative lachman test was $0.23+0.530 \mathrm{SD}$ with highly significant $\mathrm{p}$ value $<0.01$ suggesting that stability was significantly improved after double bundle ACL reconstruction. Results of anterior drawer test after double bundle ACL reconstruction were $85 \%$ (34cases) were negative. The mean of pre-operative ADT was $2.40+0.672$ $\mathrm{SD}$ and post-operative mean $0.2+0.516$ with significant $\mathrm{p}$ value $<0.01$. Results of Pivot shift test after double bundle ACL reconstruction were $72.5 \%$ (29 cases) were negative. The mean preoperative PST was $2.10+0.632$ SD and postoperative was $0.35+0.622$ with highly significant $\mathrm{p}$ value < 0.01 suggesting that there was highly significant improvement in the stability after double bundle ACL reconstruction. T. muneta M.D., PhD H. Koga, M.D. et al. (2007) ${ }^{[14]}$ in their series manual knee laxity testing revealed that negative Lachman and Pivot shift test results in more patient in double bundle group than in single bundle group. Post operatively they had statistically greater number of patients in single bundle group had positive finding based on the Lachman test (21\% in the single bundle group and only $3 \%$ in double bundle group) and pivot shift test - $41 \%$ in the single bundle group and $15 \%$ in the double bundle group. Kazunori Yasuda, M.D., Phd. D. Eiji Kondo, M.D., Hiroki Ichiyama, M.D. et al. ${ }^{[15]}$ in their series regarding the post-operative manual knee laxity test, positive Lachman test were found in 4 patients (7.01\%), all were evaluated as + and none was evaluated as ++ , Positive pivot shift test were detected in only one patient (1.7\%). Kazunori Yasuda, M.D., Phd. D. et al. ${ }^{[15]}$ In their series regarding pivot shift test $9(37.5 \%)$ patients were evaluated as + and 3 patients $(12.5 \%)$ were evaluated as ++ in the single bundle group while $3(12.5 \%)$ patients were evaluated as + , and no patient was evaluated as ++ in the double bundle group. Masayoshi Yagi, Ryosuke Kuroda, M.D., et al. ${ }^{[16]}$ in their series the Lachman test revealed positive results in two (10\%) and three (15\%) patients in the anteriomedial group and Posterolateral reconstruction group respectively, while no $(0 \%)$ patient in double bundle group showed positive instability. Muneta $\mathrm{T}$, koga $\mathrm{H}$, Morito $\mathrm{T}$, Yagishita K. (2006) ${ }^{[17]}$ In their series post-operative results of manual knee laxity tests. A statistically greater number of patients in the single bundle group were positive based on the Lachman test (34\% in the single bundle group and $13 \%$ in the double bundle group, respectively).

No major complications were seen in our series except superficial infection in 3 cases (7.5\%). 3 cases $(7.5 \%)$ had difficulty in regaining the motion. There were no cases in our series which had graft avulsion, Graft impingement. There were 3 cases $(7.5 \%)$ of superficial infection which were all treated and responded well to intravenous antibiotics. The average tourniquet time in our series was 1 hour 10 minutes and here were no case of tourniquet palsy in our series. Yasuda et al. ${ }^{[15]}$ in their series, no major complication were recorded at a minimum follow up of 24 months, and they suggested that anatomical double bundle ACL reconstruction appears to be a safe and practicable technique. Kazunori Yasuda, M.D., Phd. D. Eiji Kondo, M.D., Hiroki Ichiyama, M.D. et al. (DEC.2004) ${ }^{[15]}$ in their series they did not experience Any intraoperative problems concerning tunnel positioning, the graft placement, or the graft fixation. There were no post- operative complication, such as infection, neurovascular injury, cartilage injury, injuries in other knee structures or delayed wound healing. T muneta M.D., Ph. D H. Koga, M.D. et al. (2007) ${ }^{[14]}$ in their series there were no problematic loss of knee motion in either the single bundle or double bundle group. Prolonged joint inflammatory reaction that continued for more than 3 months after the surgery or that occurred at more than 3 months postoperatively was found in $4(11 \%)$ patients in the single bundle group and $2(5.5 \%)$ patients in double bundle group.

\section{Conclusion}

Most of patient who underwent double bundle ACL reconstruction were satisfied with the results and achieved pre-injury status in our study. Reconstructed ACL (AM and PL bundles) in anatomic ACL reconstruction recreates normal anatomy and similar biomechanical properties as native ACL. Double bundle ACL reconstruction provides anterior and rotational stability. This Anatomical ACL reconstruction is a good technique for the ACL reconstruction in athlete and high demanding patients who has to undergo pivoting most often in their life time.

\section{References}

1. Bonnet A. Traité des maladies articulaires 2ème édition Bailliére, Paris 1853,354-357.

2. Duthon VB, Barea C, Abrassart S, Fasel JH, Fritschy D, Ménétrey J. Anatomy of the anterior cruciate ligament. Knee SurgSports Traumatol Arthrosc 2006;14:204-213.

3. Hwang MD, Piefer TR, Lubowitz JH. ACL footprint 
Anatomy: Systemic review of the 21 st century literature. Arthroscopy 2012;28:728-734.

4. Augustine RW. The unstable knee. Am J Surg 1956;92:380-388.

5. Eck CF, Lesniak BP, Schreiber VM, Fu FH. Anatomic single- and double-bundle anterior cruciate ligament reconstruction flowchart. Arthroscopy 2010;26-2:258268. [PubMed].

6. Ganesh G, Redfern J, Greis P, Burks R. Revision anterior cruciate ligament reconstruction. Am J Sports Med 2011;39(1):199-217.

7. Marchant B, Noyes F, Barber-Westin S, Fleckenstein C. Prevalence of nonanatomical graft placement in a series of failed anterior cruciate ligament reconstruction. Am J Sports Med 2010;38(10):1987-1996.

8. Allen CR, Giffin JR, Harner CD. Revision anterior cruciate ligament reconstruction. Orthop Clin North Am 2003;34-1:79-98. doi: 10.1016/S0030-5898(02)00066-4. [PubMed] [Cross Ref].

9. Harner CD, Giffin JR, Dunteman RC, Annunziata CC, Friedman MJ. Evaluation and treatment of recurrent instability after anterior cruciate ligament reconstruction. Instr Course Lect 2001;50:463-474. [PubMed].

10. Brig Pathania VP, Col LT, Joshi GR et al. Anterior cruciate ligament reconstruction with BPTP through mini arthrotomy. MJAFI 2004;60:15-19.

11. Muneta T, Seiya I, Yagishita K, Ogiuchi T, Yamamoto $\mathrm{H}$, Shinomiya K. Two bundle anterior cruciate ligament using semitendinosus tendon using endobutton. Operative technique and preliminary results. Arthroscopy 1999;15:618-623.

12. Christian Latterman, MD, Boris Zelle A, Mario Ferretti MD, Anikar Chhabra MD, Freddie MD Hfu. Anatomic Double bundle anterior cruciate ligament Reconstruction. Technique in Orthopeadics 2005;20(4);414-420.

13. Johnson AJ, Erikson E, Haggmark T, Pope MH. Five to ten years follow - up evaluation after the reconstruction of anterior cruciate ligament. Clin Orthop 1994;83:122140.

14. T Muneta MD, Ph.D H Koga MD et al. A prospective study of - strand semitendinosus tendon anterior cruciate ligament reconstruction comparing single bundle and double bundle technique. Journal or Arthroscopic and Related Surgery, (June) 2007;23:618- 628.

15. Kazunori Yasuda MD, Phd. D. Eiji Kondo MD, Hiroki Ichiyama, MD et al. Anatomical reconstruction of the anteriomedial and posteriolateral bundles of the ACL using hamstring tendon graft. Journal or Arthroscopic and Related Surgery 20(10),1015-1025.

16. Masayoshi Yagi, Ryosuke Kuroda MD et al. Double bundle anterior cruciate ligament reconstruction can improve rotational stability. Clinical Orthopeadics and Related Research 2006;454:100-107.

17. Muneta $\mathrm{T}$, Koga $\mathrm{H}$, Morito $\mathrm{T}$, Yagishita $\mathrm{K}$. Retrospective study of midterm outcome of two bundle anterior cruciate ligament reconstruction using quadrupled semitendinosus tendon in comparison with double bundle reconstruction. Arthroscopy 2006:22:252258 . 